\title{
EQUIDAD EN EL ACCESO AL CONOCIMIENTO A TRAVÉS DE LAS TIC, LA EXPERIENCIA DE LA MODALIDAD ABIERTA Y A DISTANCIA DE LA UTPL A TRAVÉS DE LA RED DE AULAS VIRTUALES
}

\author{
(EQUITY IN THE ACCESS TO KNOWLEDGE THROUGH TIC, THE EXPERIENCE OF OPEN- \\ DISTANCE EDUCATION IN UTPL THROUGH VIRTUAL CLASSROOMS NETWORK)
}

\author{
Verónica Condór Bermeo y Elsa Cárdenas Sempertegui \\ Universidad Técnica Particular de Loja, UTPL (Ecuador)
}

\section{RESUMEN}

Durante el año 1999, se inicia el proceso de transformación de la modalidad abierta y a distancia de la UTPL, al incorporar las TIC en el proceso de enseñanza-aprendizaje, para lo cual se realiza una alianza estratégica con la empresa Globatel, proveedora para América del Sur de la plataforma tecnológica necesaria para la creación de Aulas Virtuales con capacidad interactiva a distancia, se procede a la instalación de la Red de Aulas Virtuales en un total de 20 centros universitarios de la UTPL, en 20 ciudades del Ecuador.

A la alianza entre Globatel y la UTPL en enero de 2001, se incorpora la Red Global de Aprendizaje para el Desarrollo patrocinada por el Banco Mundial y presente en varios continentes, constituyéndose en la red de mayor cobertura dentro de un país de todos los pertenecientes a esta red y siendo la pionera en el esfuerzo para hacer llegar a los lugares alejados de las grandes ciudades las posibilidades de ofrecer educación de calidad.

El desarrollo de las TIC nos hace pensar sobre el uso adecuado que se les debe dar a estas herramientas y, sobre todo, en el campo de la educación como elemento básico para lograr la equidad y calidad en el acceso al conocimiento. Sobre esta perspectiva se desarrolla el presente trabajo para lo cual analizamos la experiencia de la Red de Aulas Virtuales de la UTPL.

\section{ABSTRACT}

During 1999 it began the process of transformation into open-distance education in UTPL, adding the TIC in the teaching-learning process. In order to accomplish this, a strategic alliance was made with Globatel. A Virtual Classrooms Network was installed in a total of 20 universities, in 20 cities in Ecuador.

The Global Network of Learning for Development joins the partnership Globatel-UTPL in January 2001. The Global Network is sponsored by World Bank. The parnership became the holder of the network with the largest range in a country. 
The development of the TIC makes us think about the appropiate use of such tools, specially in the education field as a basic component to achieve equity and quality in the access to knowledge. The following work is based on this perspective in which the Virtual Classrooms Network experience has been analized.

\section{DESARROLLO}

La equidad en el acceso al conocimiento en un mundo globalizado, usando como herramientas a las TICy espeć́ficamente a la Red de Aulas Virtuales de la UTPL, es el tema de este trabajo. La equidad cobra vigencia al momento de analizar la brecha digital que se genera por la existencia de una minoría conectada a Internet y la mayoría marginada de este medio. Para apoyar a que esta brecha sea cada vez más corta debemos enfocar el máximo aprovechamiento en la nueva era tecnológica para brindar buenos resultados a todas las personas en el Ecuador sin exclusión de ningún tipo.

Lo más importante a destacar del desarrollo de las TIC es que se amplía el ámbito en que la humanidad puede emplear las aplicaciones tecnológicas para erradicar la pobreza, esto implica la conciencia y el respectivo esfuerzo para velar porque la humanidad tenga acceso a esa potencialidad y no solo unos pocos afortunados. (Informe Desarrollo Humano, PNUD, 2001).

Las innovaciones tecnológicas afectan doblemente al desarrollo humano. En primer término, elevan de modo directo la capacidad humana. En segundo lugar, constituyen un medio para lograr el desarrollo humano debido a sus repercusiones en el crecimiento económico gracias al aumento de la productividad que generan. Crean así mismo nuevas actividades e industrias, como el sector de la tecnología de la información y las telecomunicaciones que contribuyen al crecimiento económico y a la creación de empleos.

Para la utilización de las TIC en los sistemas educativos, se considera que son necesarias estructuras de educación y formación más variadas, abiertas y flexibles, que constituyan una opción capaz de multiplicar y diversificar las ofertas educativas para todas las personas, ayudando a realizar el ideal de la verdadera democratización de la educación. Esto implica que se potencien las modalidades de enseñanza abierta y a distancia con la utilización conjunta de los materiales educacionales y de los avances tecnológicos de la telecomunicación y de la informática.

En este sentido, considerando los desafíos que la educación tanto en los países desarrollados como en vías de desarrollo, no hay que sorprenderse de que la educación a distancia sea considerada como una nueva e importante estrategia que podría hacer una contribución definitiva para la solución de los problemas de acceso, calidad y equidad. (UNESCO, 1999). 


\section{LA EXPERIENCIA DE LA UTPL}

La Modalidad Abierta y a Distancia de la Universidad Técnica Particular de Loja inicia sus actividades en octubre de 1976 con 1266 estudiantes. El modelo desde el cual partió fue el de la UNED de España, pero con las adaptaciones propias de las características del Ecuador.

Los recursos tienen su origen en la Universidad ya existente en modalidad presencial, con una característica muy poco usual de las provincias de mayor auge económico y comercial o con mejores condiciones de comunicación, sino en una provincia de difícil acceso y comunicaciones deficientes, es decir, con difícil perspectiva de crecimiento o de mejora de las comunicaciones por tierra. (Barbosa, 2001).

En sus inicios la comunicación era bidireccional a través de correspondencia, teléfono, fax, las tutorías presenciales de computación, inglés en los centros universitarios, así como en algunas ocasiones los estudiantes se trasladaban a la sede de la UTPL en Loja.

Durante el año 1999, se inicia el proceso de transformación de esta modalidad, para el máximo aprovechamiento de las nuevas tecnologías de la información y de la comunicación se realiza una alianza estratégica entre la UTPL y la empresa Globatel, proveedora para América del Sur de la plataforma tecnológica necesaria para la creación de Aulas Virtuales con capacidad para la educación y capacidad interactiva a distancia, se procede desde el año 1999 a la instalación de la Red de Aulas Virtuales en un total de veinte centros universitarios de la UTPL, correspondientes a veinte ciudades del Ecuador. En la primera fase se incorporan 6 aulas situadas en las ciudades de Loja, Quito, Guayaquil, Ibarra, Cuenca y Santo Domingo. En estas localidades se instalaron bajo el sistema Train Net, que consiste en aulas con una computadora por persona desde el cual el participante puede interactuar con el profesor a través de un programa diseñado especialmente para ese fin.

En el segundo semestre del año 2000, se inicia la segunda fase con la incorporación de ocho aulas en las ciudades de Esmeraldas, Manta, Portoviejo, Machala, Tulcán, Latacunga, Ambato y Riobamba y se incorpora un rediseño del modelo de interactuación, se establecen aulas más parecidas al salón de clases y auditorios en los cuales pueda llegar la capacitación a un número mayor de participantes. En el 2002 se incorporan las ciudades de Macas, Tena, Zamora, éstas en la amazonía ecuatoriana y Santa Cruz en las Islas Galápagos.

La misión de la Red de Aulas Virtuales es difundir programas de capacitación continua y formal, en primera instancia a los estudiantes dela Modalidad Abierta y a Distancia y al público en general identificados por sectores sociales, sean estos públicos o privados, utilizando tecnologías de telecomunicación y multimedias. (AULAS VIRTUALES UTPL, 2004). 


\begin{tabular}{|l|c|}
\hline \multicolumn{1}{|c|}{ REGIÓN } & $\begin{array}{c}\text { Capacidad insta- } \\
\text { lada }\end{array}$ \\
\hline \multicolumn{1}{|c|}{ REGIÓN SIERRA } & 60 \\
\hline AMBATO & 700 \\
\hline LOJA & 90 \\
\hline CUENCA & 30 \\
\hline TULCÁN & 350 \\
\hline QUITO & 40 \\
\hline GUARANDA & 40 \\
\hline RIOBAMBA & 60 \\
\hline IBARRA & 70 \\
\hline LATACUNGA & 50 \\
\hline STO DOMINGO & \\
\hline \multicolumn{1}{|c|}{ REGIÓN COSTA } & 50 \\
\hline PORTOVIEJO & 60 \\
\hline QUEVEDO & 40 \\
\hline MANTA & 120 \\
\hline GUAYAQUIL & 60 \\
\hline MACHALA & 40 \\
\hline ESMERALDAS & \\
\hline REGIÓN ORIENTAL & 60 \\
\hline PUYO & 50 \\
\hline TENA & 28 \\
\hline MACAS & 60 \\
\hline ZAMORA & 20 \\
\hline NUEVA LOJA & 2070 \\
\hline REGION INSULAR & \\
\hline SANTA CRUZ & \\
\hline TOTAL & \\
\hline & \\
\hline
\end{tabular}

Fuente: Aulas Virtuales UTPL. Elaboración propia.

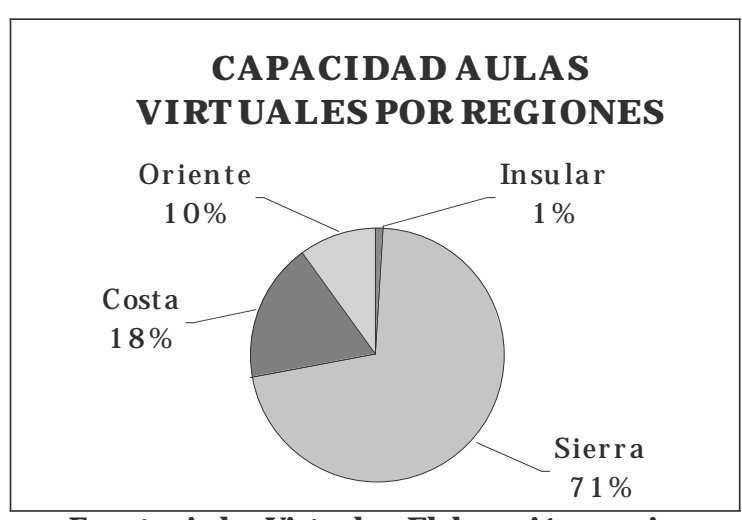

Fuente: Aulas Virtuales. Elaboración propia. 


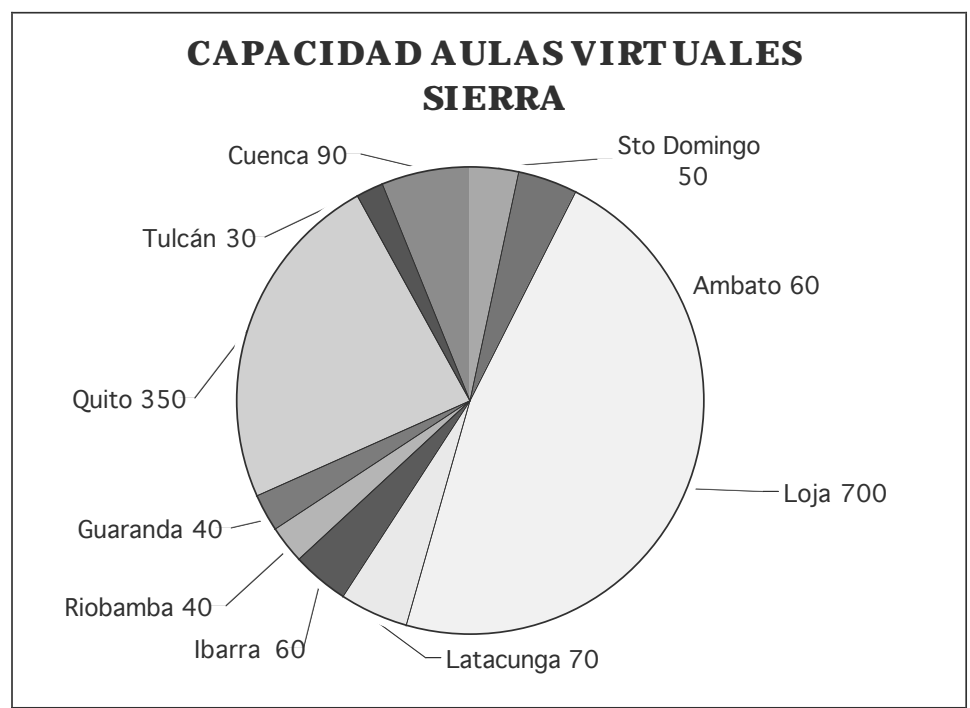

Fuente: Aulas Virtuales. Elaboración propia.

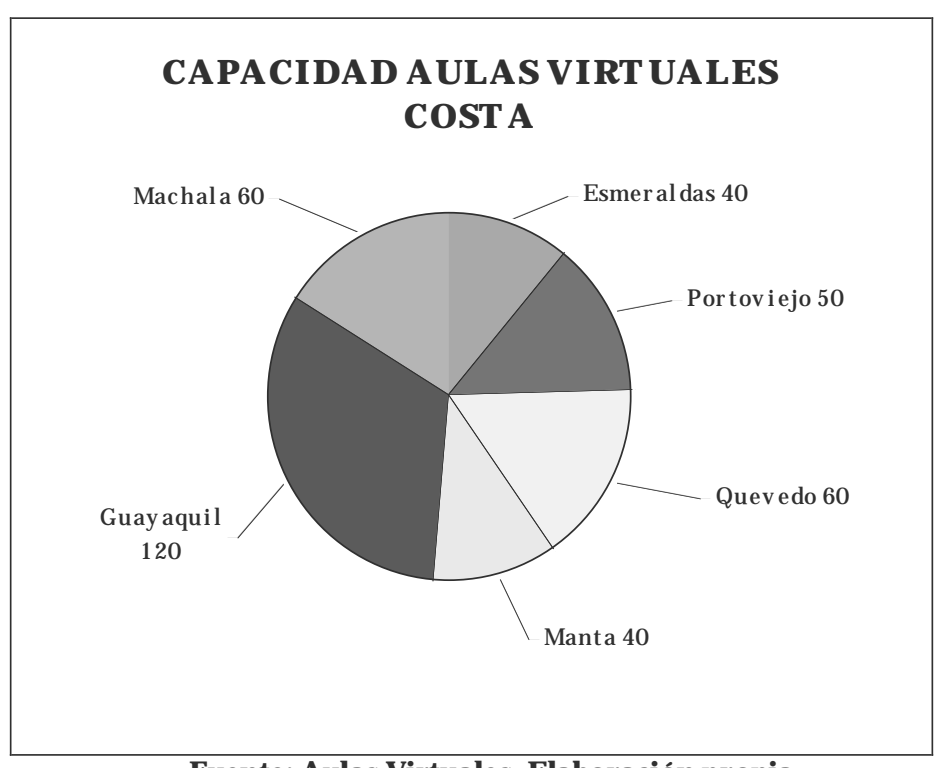

Fuente: Aulas Virtuales. Elaboración propia.

A la alianza entre Globatel y la UTPL en enero de 2001, se incorpora la Red Global de Aprendizaje para el Desarrollo patrocinada por el Banco Mundial y presente ya en varios continentes, constituyéndose en la red ecuatoriana de mayor cobertura dentro de todos los países pertenecientes a esta Red y siendo la pionera en el esfuerzo para hacer llegar a los lugares alejados de las grandes ciudades las posibilidades de ofrecer tecnología. Desde cada aula virtual remota los alumnos pueden: solicitar hablar con el 
profesor, registrarse para atender la lección, interactuar con sus compañeros de otras aulas remotas, preguntar, comentar y responder a los requerimientos que la clase o que el tema amerite. Actualmente los estudiantes de la UTPL reciben tutorías y conferencias sobre diferentes disciplinas y áreas del conocimiento como: salud, desarrollo urbano y rural, educación, experiencias emprendedoras, ambiente, finanzas, tecnología, gobierno, administración etc., pero, además, se ha trabajado junto a instituciones como el Ministerio de Educación en el proyecto maestr@s.com y junto al Ministerio de Comercio Exterior se desarrolló el proyecto de desarrollo de microempresas con los familiares de los ecuatorianos que han migrado a España, integrando para este proyecto a través de la Red Virtual al Centro Universitario de la UTPL en Madrid.

Cuadro 1: Cursos realizados por áreas académicas

\begin{tabular}{|l|l|l|l|}
\hline \multicolumn{1}{|c|}{ AREA } & \multicolumn{2}{c|}{ ANOS } \\
\cline { 2 - 4 } & $\mathbf{2 0 0 3}$ & $\mathbf{2 0 0 4}$ & $\mathbf{2 0 0 5}$ \\
\hline AREA ADMINISTRATIVA & & & \\
\hline Diplomado en Gerencia Estratégica del Desempeño. & 1 & 1 & \\
\hline Diplomado en Auditoria de Gestión dela Calidad & & & 3 \\
\hline Diplomado en Gerencia Estratégica de Mercadeo & & & 2 \\
\hline Diplomado en Intervención Social & & & 4 \\
\hline Comercio Exterior, Integración & 2 & 2 & 4 \\
\hline Migración & 1 & & \\
\hline Postgrado & & & \\
\hline Administración & 1 & & \\
\hline Liderazgo, Emprendimiento y Competitividad & 1 & 3 & 13 \\
\hline Economía y Finanzas & & 2 & 4 \\
\hline Capacitación empresas privadas y públicas & & 4 & 5 \\
\hline Turismo & 1 & & 2 \\
\hline Marketing & & & 5 \\
\hline Certificado Superior en Administración de Seguros & & & 4 \\
\hline Seguros & & 1 & 3 \\
\hline ÁREA BIOLÓGICA & & & \\
\hline Postgrado en Gerencia de Salud para el Desarrollo Local & 4 & 11 & 15 \\
\hline Salud & & 10 & 2 \\
\hline Biotecnología y Biodiversidad & & & 2 \\
\hline ÁREA SOCIO-HUMANISTICA & & & \\
\hline Estándares de Calidad - Educación a Distancia & & & 3 \\
\hline Educación & 1 & & 1 \\
\hline Maestría en Talentos y Creatividad & 1 & & \\
\hline Congreso TEFL Inglés & & & 4 \\
\hline Congreso IUNITAC & 1 & 1 & \\
\hline Gobernabilidad & & 1 & 4 \\
\hline Género & & & 2 \\
\hline Tutorías pregrado & 1 & & 1 \\
\hline Cultural y Religioso & 1 & 4 & 1 \\
\hline Legal & 1 & 3 & 1 \\
\hline Formación Religiosa & 2 & 3 \\
\hline Desarrollo Humano & & \\
\hline & & & \\
\hline
\end{tabular}




\begin{tabular}{|l|l|l|l|}
\hline \multirow{2}{*}{ AREA } & \multicolumn{3}{c|}{ AÑOS } \\
\cline { 2 - 4 } \multicolumn{1}{c|}{} & $\mathbf{2 0 0 3}$ & $\mathbf{2 0 0 4}$ & $\mathbf{2 0 0 5}$ \\
\hline Diplomado en Derecho Empresarial & & & 2 \\
\hline Sector público y Gobiemos locales & 1 & 2 & 5 \\
\hline Comunicación & & 1 & 1 \\
\hline ÁREA TÉCNICA & & & \\
\hline Redes & & & 5 \\
\hline Telecomunicaciones & & & 2 \\
\hline
\end{tabular}

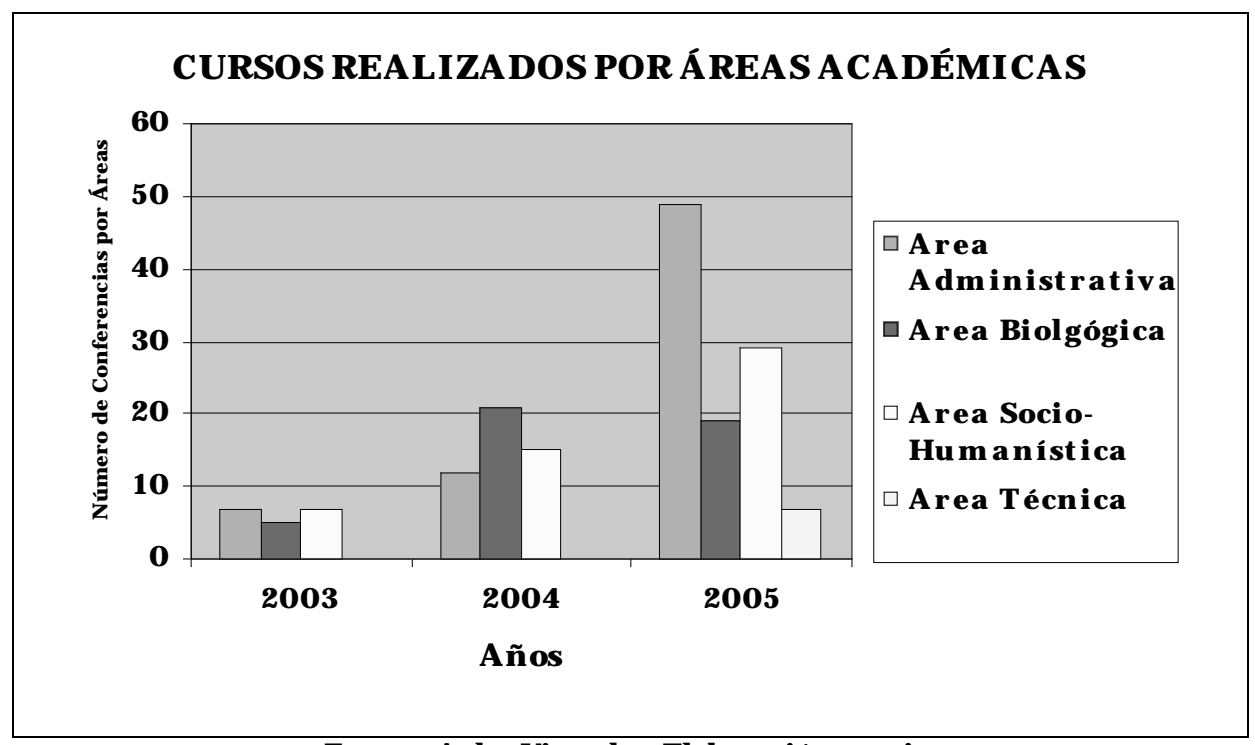

Fuente: Aulas Virtuales. Elaboración propia.

\section{CURSOS NACIONALES E INTERNACIONALES}

\begin{tabular}{|l|c|c|c|}
\hline & $\mathbf{2 0 0 3}$ & $\mathbf{2 0 0 4}$ & $\mathbf{2 0 0 5}$ \\
\hline Cursos Nacionales & 16 & 46 & 79 \\
\hline Cursos Internacionales & 2 & 1 & 19 \\
\hline
\end{tabular}

Fuente: Aulas Virtuales. Elaboración propia. 


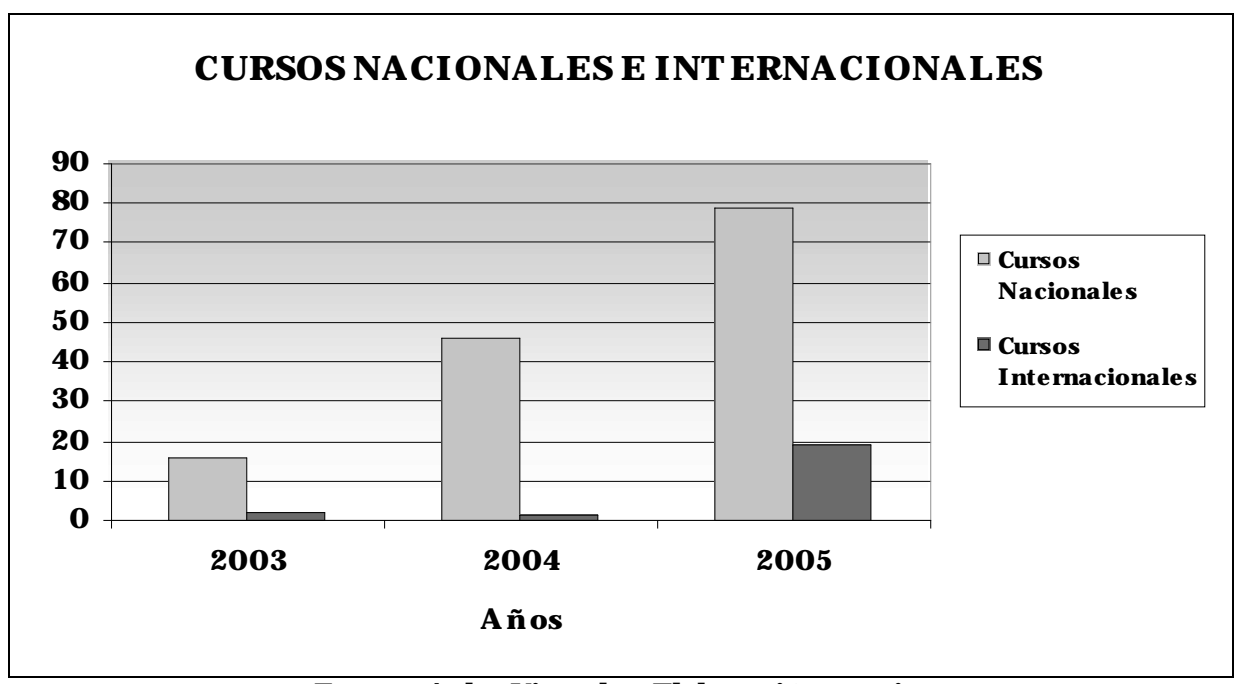

Fuente: Aulas Virtuales. Elaboración propia.

\section{ASISTENTES A LOS CURSOS}

\begin{tabular}{|l|c|c|c|}
\hline & $\mathbf{2 0 0 3}$ & $\mathbf{2 0 0 4}$ & a julio 2005 \\
\hline Número de asistentes aulas virtuales & 1587 & 7126 & 9676 \\
\hline \% Crecimiento en relación a 2003 & & $449 \%$ & $135 \%$ \\
\hline
\end{tabular}

Fuente: Aulas Virtuales. Elaboración propia.

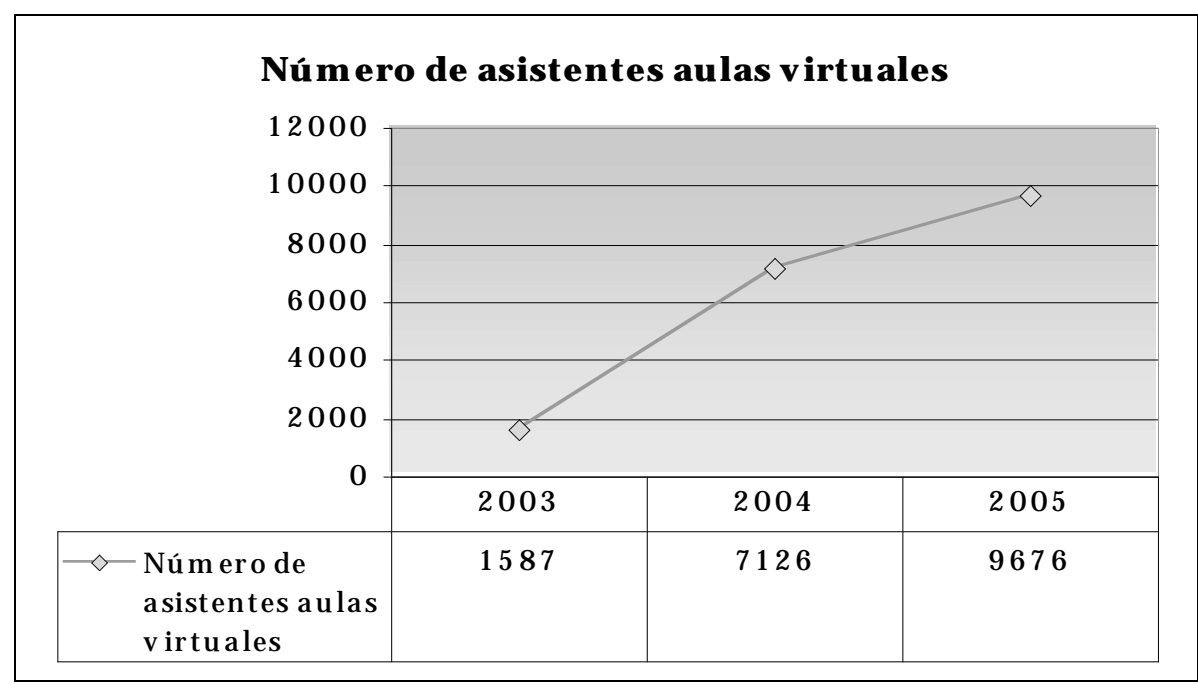

Fuente: Aulas Virtuales. Elaboración propia. 
Antes de la instalación de la Red de Aulas Virtuales, en Ecuador era imposible pensar que la Amazonía, los Andes, la Costa y las Islas Galápagos, se integren al mismo tiempo para seguir las tutorías, conferencias, debates, etc...

El compromiso de descentralización y democratización del acceso a la educación de la UTPL se ha fortalecido con la utilización de las tecnologías de la información y de la comunicación, ya que sin importar las condiciones geográficas, sociales o económicas se está permitiendo equidad en el acceso al conocimiento, con lo cual se está integrando a un país, pues todas las personas tienen las mismas oportunidades, a pesar de no tener las mismas circunstancias y trabajar por la equidad en el acceso al conocimiento, es decisivo al momento de aportar concretamente a lo que plantea la CEPAL con "la globalización de los valores", o lo que las Naciones Unidas señala en la declaración de los 8 Objetivos de Desarrollo del Milenio.

En definitiva, en la sociedad actual, la educación está llamada a producir un efecto sociocultural, a través de la redefinición de las capacidades de las personas en un contexto de equilibrio entre el progreso tecnológico, el crecimiento económico y el desarrollo social. Si en los próximos años América Latina es capaz, a través de sus sistemas educativos, de ofrecer oportunidades de aprendizaje relevantes y de calidad a la mayoría de personas, entonces quizá por primera vez no esté tan lejano ni inalcanzable avanzar en el cierre de la brecha de la inequidad y, en consecuencia, en la construcción de una sociedad más justa y más humana.

Ya lo señaló J uan Pablo II “Sea cual fuere nuestra edad, tenemos que afrontar el desafío de los descubrimientos y nuevas tecnologías, aplicándoles una visión moral basada en nuestra fe, en nuestro respeto a la persona humana y en nuestro empeño de transformar el mundo según el plan de Dios" (J UAN PABLO II).

\section{CONCLUSIONES}

- El apoyo de las TIC en los procesos académicos de pre y postgrado han permitido el diálogo didáctico mediado fundamental en el mejoramiento del proceso enseñanza- aprendizaje en educación a distancia.

- El desarrollo humano es básico en los procesos de integración, ya que al ser la educación una variable del IDH, se fortalece ésta al permitir que mayor número de personas accedan a un sistema educativo con calidad y pertinencia.

- Los involucrados en los procesos de enseñanza-aprendizaje deben actualizarse en la utilización de estas herramientas para aprovechar sus potencialidades.

- Se mejora el desempeño del expositor, optimizando el tiempo de capacitación, ya que se puede tener una conferencia simultánea en 21 ciudades del país. 
- Se minimizan los costos derivados de la dispersión geográfica, ahorro en viáticos, movilización y alojamiento del personal.

- Se debe tomar en cuenta que el porcentaje de accesibilidad de la población ecuatoriana a Internet es del 2,8\% en el 2004, lo cual refleja el escaso acceso a este medio considerando Internet como las TIC mas difundida, tomando en cuenta este dato debemos preocuparnos porque la utilización de la Red de Aulas Virtuales sea con un sentido de integración ético, dirigido hacia todos los sectores de la población para evitar el aumento de la brecha digital que da origen a nueva clasificación de inforicos e infopobres.

- Las políticas de estado con respecto a la educación deben adoptar a las TIC como un medio de integración, desarrollo y democratización de los pueblos a través de éstas, asignando recursos económicos y financieros para que sean difundidas a todas las personas, pues deben tener las mismas oportunidades a pesar de no tener las mismas circunstancias.

- Las TIC son un medio que apoya la difusión del conocimiento en un mundo globalizado, por ende los contenidos deben continuar siendo investigados y adaptados a las diferentes realidades.

- Por si sólo el acceso a las TIC no resulta en generación de conocimientos ni en la transformación de las igualdades, por tal razón se vuelve prioritario el promover su uso con sentido ético y apropiación social.

\section{REFERENCIAS BIBLIOGRÁFICAS}

Adell, J. (1997). Tendencias en Educación en la sociedad de las tecnologías de información. Revista Electrónica de Tecnología Educativa. España.

Barbosa, J. (2001). La Información es un componente que hay que saber seleccionar. Artículo publicado en el Informe del Desarrollo Humano del PNUD 2001. PNUD Ecuador

Garcia Guadilla, C. (1992) Conocimiento, Educación Superior y Sociedad en Amé rica Latina.
Kliksberg, B. (2002). Ética y Desarrollo, la relación marginada. Editorial El Ateneo y BID. Buenos Aires, Argentina

PNUD (Programa de las Naciones Unidas para el Desarrollo) (2001). Informe sobre Desarrollo Humano. Las Tecnologías de información y comunicación para el desarrollo humano. PNUD, Ecuador.

Silvio, J. (2000). La Virtualización de la Universidad. IESALC Caracas, Venezuela. 


\section{PALABRAS CLAVE}

Equidad, desarrollo, tecnología, acceso, información, conocimiento.

\section{KEY WORDS}

Equity, development, tecnology, internet access, information, knowledge.

\section{PERFIL ACADÉMICO DE LAS AUTORAS}

Verónica Condor Bermeo:

- Economista.

- $\quad$ Diplomado en Gerencia Estratégica de Mercadeo.

- $\quad$ Diplomado Superior en Fundamentos de la Educación a Distancia e Investigación.

- Especialista en Planificación y Gestión de Proyectos de Cooperación Internacional para la Educación, la Ciencia y la Cultura. Organización de Estados Iberoamericanos (OEI).

- Especialista en Planificación Curricular y Organización de Sistemas de la Educación a Distancia.

- Maestrante en Educación a Distancia.

- E-mail: vpcondor@utpl.edu.ec.

Elsa Cárdenas Sempertegui:

- $\quad$ Licenciada en Contabilidad y Auditoría.

- $\quad$ Peritaje en Inglés.

- Doctora en Contabilidad y Auditoría.

- Diplomado en Intervención Social.

- $\quad$ Diplomado en Gerencia Estratégica de Mercadeo.

- $\quad$ Diplomado en Control Total de Calidad en Institudciones Educativas.

- Diplomado Superior en Fundamentos de la Educación a Distancia e Investigación.

- Especialista en Planificación Curricular y Organización de Sistemas de la Educación a Distancia.

- Maestrante en Educación a Distancia.

- E-mail: ebcardenas@utpl.edu.ec.

Dirección postal: $\quad$ Apartado Postal: 1101608.

Fecha recepción del artículo: 21. 02. 2006

Fecha aceptación del artículo: 30. 02. 2006 